Acta Universitatis Wratislaviensis No 3742

Studia Linguistica XXXV Wrocław 2016

DOI: $10.19195 / 0137-1169.35 .7$

\title{
INES BOSE
}

Martin-Luther-Universität Halle-Wittenberg, Deutschland

\author{
KATI HANNKEN-ILLJES
}

Philipps-Universität Marburg, Deutschland

\section{Wie Vorschulkinder Geltung etablieren}

Das Geben von Gründen kann verschiedene Funktionen haben: die Bearbeitung von Dissens, die Problemlösung, aber auch die Etablierung von gemeinsamem Wissen und damit die Etablierung von Geltung. Zur dieser letzten, epistemischen Funktion von Argumentation, der Etablierung von Geltung im Gespräch durch das Geben von Gründen, gibt es nur wenige Arbeiten, dies gilt besonders für Gespräche unter kleinen Kindern. Wir untersuchen in einer Longitudinalstudie Begründungshandeln in natürlicher Kommunikation unter Vorschulkindern (3 - 7 Jahre), mit dem die Kinder Geltung etablieren und argumentative Ressourcen erwerben. Argumentative Ressourcen verstehen wir als Etablierung dessen, was Kinder später in der Argumentation als Gründe anführen können, und knüpfen damit an die Ethnographie der Argumentation an (Krummheuer 1995, Prior 2005, Hannken-Illjes et al. 2007). Unsere Forschung zielt auf eine integrierte (multimodale) Beschreibung und Analyse sprachlicher, stimmlich-artikulatorischer und körperlicher Praktiken. Konzeptionell verknüpfen wir dabei interaktionslinguistische, (ausdruckspsychologisch-)sprechwissenschaftliche und (rhetorisch-)argumentationswissenschaftliche Konzepte. Methodisch wählen wir einen deskriptiv-qualitativen, gesprächsanalytisch und ethnomethodologisch informierten Zugriff. Die Datenbasis bilden zwei voneinander unabhängige Langzeit-Videokorpora authentischer (Spiel-)Gespräche zwischen miteinander vertrauten Vorschulkindern über jeweils drei Jahre im häuslichen Umfeld sowie im Kindergarten: Korpus A (19931998) und Korpus B (2014-2017), insgesamt ca. 120 h, Altersspanne in beiden Korpora jeweils 3.0 bis 7.0 Jahre.

Folgende Forschungsfragen sind für uns leitend: Wie etablieren Vorschulkinder in der Kommunikation untereinander Geltung durch Begründungshandeln und schaffen so die Basis für Kooperation? Welchen Status und welche Funktion haben stimmlicher und körperlicher Ausdruck innerhalb dieser Verfahren und in welchem 
Verhältnis stehen sie zu ersten Formen der Argumentation? Lassen sich über den Beobachtungszeitraum hinweg Entwicklungen in der Fähigkeit zur Etablierung von Geltung aufzeigen (z.B. in der Verwendung von Topoi)? Im Folgenden wollen wir einige Ansätze des Forschungsprojekts skizzieren und erste Analysen präsentieren.

\section{Die Etablierung von Geltung durch Argumentieren}

Wir verstehen Argumentieren im Sinne einer empirisch rekonstruierbaren kommunikativen Praktik im situativen Kontext (vgl. u.a. Deppermann 2003, Quasthoff/Kern 2007, Heller 2011, Arendt 2015). Argumentation wird in der Regel als eine zentrale kommunikative, kulturgebundene Praxis aufgefasst (u.a. Johnstone Jr. 1965, Kopperschmidt 1989, Habermas 1995, Spranz-Fogasy 2003), die durch Begründungshandeln die Möglichkeit der Einflussnahme auf den Anderen bietet, eigene Überzeugungsbereitschaft impliziert und so Kooperation und gemeinsames Handeln ermöglicht. Damit ist Argumentation thematisiert als ein ,in unserer Kultur weitverbreitetes Verfahren zur Klärung unklarer oder strittiger Sachverhalte“" (Spranz-Fogasy 2003:28): Gesprächsteilnehmer/innen argumentieren demnach dann, wenn das Gesprächshandeln ins Stocken geraten ist. Voraussetzung für Argumentation ist in dieser Auffassung ein verbal markierter Dissens bzw. eine Unklarheit - „die praktische Gültigkeit einer Darstellung ist gesprächslokal in Frage gestellt und muss her- bzw. wieder hergestellt werden" (ebd.:31). Dissens, ob latent oder aktuell, macht Argumentation, also das Geben und Einfordern von Gründen in Bezug auf einen strittigen Punkt (Hannken-Illjes 2004:14), zu einem möglichen, wenn nicht erwartbaren kommunikativen Verfahren. Hier dient Argumentation dazu, einen strittig gewordenen Geltungsanspruch durch das Geben von Gründen zu bearbeiten, mit dem Ziel, dass die Beteiligten zum Abschluss der Argumentation von einer gemeinsamen Geltungsbasis aus weiterhandeln können.

Durch Argumentieren wird versucht, eine (potenziell) strittige Aussage durch eine schon geltende zu stützen. Damit wird Argumentation aber immer auch zu einem „Reden über die Geltungsbedingungen von Äußerungen“ (Kopperschmidt 1989:28). Das Geben von Gründen dient hier der Etablierung und Sicherung einer gemeinsamen Geltungsbasis. Eine Argumentationsanalyse erlaubt also (immer auch) eine Analyse zweier epistemischer Bedingungsgefüge: dessen, was von den Gesprächsteilnehmer/inne/n für geltend gehalten wird, und dessen, von dem sie annehmen, dass es auch das Gegenüber für geltend halten muss. Diese epistemische Dimension der Argumentation ist vor allem in der Theorie zur Topik ausgearbeitet worden (u.a. Conley 2000, Knoblauch 2000, Hannken-Illjes et al. 2007, Schwarze 2010). Topoi haben insofern eine Entlastungsfunktion, als sie für alle Diskursteilnehmer/innen als geltend behandelt werden. Für Knoblauch (2000:653) überschneidet sich die Topik damit ,deutlich mit dem, was innerhalb der modernen Soziologie als ,Wissen" bezeichnet wird“. 
Mit Blick auf unsere Daten scheint uns dieser Aspekt insbesondere für die Argumentation in Kind-Kind-Kommunikation des Vorschulalters wichtig, er ist bisher aber kaum bearbeitet. Erste Datenanalysen unserer Korpora (s. unten) zeigen, dass Vorschulkinder im gemeinsamen Spiel Begründungshandeln oft nicht zur Dissensbearbeitung einsetzen, sondern kooperativ-kollaborativ zur gemeinsamen Erschaffung von Spielwelten und zur Ausstellung von Konsens über eingebrachte (Spiel-)Ideen. Argumentieren in der Kind-Kind-Kommunikation hat also auch eine epistemische Dimension - das Geben von Gründen zur Etablierung und Sicherung einer gemeinsamen Geltungsbasis.

Diese Zusammenführung des Argumentationsbegriffs mit der kollaborativen Entwicklung von Geltung ohne lokalen Dissens mag auf den ersten Blick kontraintuitiv wirken, denn aus rhetorischer Perspektive dient Argumentation dazu, das Gegenüber zu überzeugen oder die eigene Position zu schärfen. Für uns ist jedoch eine Rhetorikkonzeption leitend, die das Konzept der Persuasion durch das Konzept der Identifikation ergänzt (z.B. Burke 1969). D.h., rhetorisches Handeln ist nicht nur durch Agonalität charakterisiert, sondern zielt auch auf die Identifikation der Teilnehmer/innen miteinander.

Unsere Forschung verortet sich also in einem Grenzbereich der Argumentationsforschung, der Untersuchung der epistemischen Funktion von Begründungshandeln. Wenn wir im Folgenden Argumentation eng an die Etablierung von Geltung knüpfen, so folgen wir damit einem Verständnis, das auch in W. Kleins Definition (1980:19) zum Ausdruck kommt: Argumentation als die „Überführung von etwas kollektiv Strittigem in etwas kollektiv Geltendes mit Hilfe von kollektiv Geltendem“. Begründungen in argumentativem Austausch beziehen sich immer auf Aussagen (ob in Form von Schlussregeln oder Topoi), die als akzeptiert betrachtet werden. Dieses kollektive Geltende, das in der Argumentation zentral ist, um Akzeptanz für eine noch strittige Aussage zu erhalten, beschreibt die epistemische Dimension von Argumentation. Durch Argumentation wird so immer wieder aktualisiert, was als geltend behandelt wird. Damit hat Argumentation immer zwei Funktionen: zum einen die Bearbeitung von Dissens (durch Auflösen oder auch Schärfen), zum anderen die Etablierung dessen, was von den Interaktionsteilnehmer/inne/n als geltend betrachtet wird.

\section{Argumentieren in kindlicher Kommunikation}

\subsection{Protorhetorik}

Das Interesse an den Möglichkeiten der Einflussnahme auf die Umwelt und vor allem auf den Anderen mit Hilfe von Sprache und anderen symbolischen Mitteln ist nicht nur ein linguistisches, sondern zugleich ein rhetorisches, in Bezug auf 
Begründungshandeln unter Kindern im Sinne einer Protorhetorik. Antos (1985) versteht unter „Protorhetorik“ eine Vorform rhetorischer Kommunikation und macht dies beispielsweise daran fest, dass Kinder bereits früh sprachliche und sprecherische Strategien nutzen, um auf ihre Umgebung Einfluss zu nehmen, und dabei auch rhetorische Figuren wie Ellipsen, Paraphrasen, Anakoluthe ohne Rhetorikausbildung mühelos verwenden. Die Verwendung sprachlich-kommunikativer Mittel ist nach Antos (1985:9) dann als (proto-)rhetorisch zu kennzeichnen, wenn es Anzeichen für eine reflektierte Verwendung gibt. Kinder eignen sich nicht protorhetorische Fähigkeiten ,,an sich“ an, sondern sie erwerben kommunikative Mittel zur Erreichung ihrer Ziele im interaktiven Handeln. In diesem Verständnis sind dann auch protorhetorische Fertigkeiten erworben und gelernt, aber noch nicht theoretisch überformt. Damit sind protorhetorische Fähigkeiten, auch Argumentation, Teil der sich entwickelnden allgemeinen kommunikativen Kompetenz (so auch Bose/Hannken-Illjes 2012). Es handelt sich um einen komplexen Erwerbsprozess, über den sich zumindest fürs Deutsche nach wie vor noch keine gesicherten Aussagen machen lassen. So ist z.B. noch ungeklärt, ob sich protorhetorische Fähigkeiten eher kontinuierlich entwickeln oder vor allem individuell unterschiedlich und z.T. diskontinuierlich (vgl. z.B. Ehlich/Bredel/ Reich 2008a:24ff.). Hierzu können vor allem repräsentative Langzeitstudien zum Vorschulalter Auskunft geben, die erst seit kurzem durchgeführt werden (z.B. auch Arendt 2014, 2015).

Kleine Kinder erleben insbesondere im Bereich der diskursiv-pragmatischen Basisqualifikationen (Ehlich/Bredel/Reich 2008a, b) Selbstwirksamkeit dadurch, dass sie durch sprachlich-kommunikatives Handeln auf ihre Umwelt einwirken, also Verhaltens- oder Einstellungsveränderungen bei anderen erlangen können. Dies bestätigen sozialpsychologische Studien zur kindlichen Argumentation (z.B. Kuhn/Udell 2003, Crowell/Kuhn 2014), die argumentativen Fähigkeiten eine zentrale Rolle für die intellektuelle Entwicklung von Kindern zubilligen. Die Entwicklung der Fähigkeit zu argumentieren hängt von der Entwicklung der Theory of Mind ab (Nelson 1996, Röska-Hardy 2011, Andresen 2005, Brüne/Brüne-Cohrs 2006 und zur geteilten Intentionalität (Tomasello 2002, Yánéz 2015). Diese Theorie des Geistes ist die Voraussetzung, um das für geltend Gehaltene des Gegenübers antizipieren zu können.

\subsection{Argumentieren von Vorschulkindern}

Auch wenn die meisten Studien davon ausgehen, dass voll entwickelte Argumentation im Sinne einer Integration von Argumentation und Gegenargumentation (u.a. Crowell/Kuhn 2014, Muller-Mirza/Perret-Clermont/Tartas 2009) erst im Schulalter ausgebildet wird, so ist doch gerade in der Argumentation eine Entwicklungsperspektive sinnvoll. Crowell/Kuhn (2014) stellen in Rückgriff auf 
Resnick/Michaels/O'Connor (2010:172) fest: „the ability and disposition to engage in reasoned discourse develops through socialization. That is, only through sustained participation in activities and environments that ,expect such behavior, support it, and reward it in overt and subtle ways"“ (Crowell/Kuhn 2014:365, in diesem Sinn auch Ehlich/Bredel/Reich 2008a:25ff.). Zugleich zeigen verschiedene Studien, dass auch bei Vorschulkindern schon argumentative Verfahren auszumachen sind (z.B. Völzing 1978, Miller 1980, J. Klein 1985, Komor 2010, Arendt 2015).

Die Erforschung von Argumentation bei Kindern im Allgemeinen hat in den letzten Jahren wieder an Aufmerksamkeit gewonnen (u.a. Kraft/Meng 2007, Brumark 2007 und 2008, Muller-Mirza et al. 2009, Pontecorvo/Arcidiacono 2010, Komor 2010, Quasthoff/Krah 2015, Arendt/Heller/Krah 2015, Perret-Clermont et al. 2015). Die meisten dieser Studien orientieren sich an der Bearbeitung von Dissens und konzentrieren sich fast ausschließlich auf die verbale Ebene der Kommunikation. Dies verschließt aus unserer Sicht insbesondere in der Untersuchung des Begründungshandelns im Vorschulalter wichtige kommunikative Praktiken.

\subsection{Geltungsetablierende Funktion kindlicher Äußerungen}

Nur wenige Studien richten das Augenmerk auf die geltungsetablierende Funktion von Begründungen und Argumentation unter Kindern, sie sind für unser Projekt aber hochinteressant. Sandoval/Cam (2010) untersuchen wissensorientierte Argumentation, indem sie Kinder im Alter zwischen acht und zehn Jahren darum bitten, zwei Figuren in einer Geschichte dabei zu helfen, die besten Gründe für die Akzeptanz einer Aussage zu finden. Die Kinder präferieren dabei Begründungen, die auf Evidenz beruhen, Autoritätsargumente gelten ihnen dagegen als am wenigsten glaubwürdig. Die Arbeit von Sandoval/Cam (2010) ist exemplarisch für Arbeiten, die vor allem an der epistemischen Funktion von Argumentation (häufig in Verbindung mit wissenschaftlicher Argumentation) interessiert sind. Ein anderes Beispiel für diese Auffassung von Argumentation ist die Arbeit von Perret-Clermont et. al. (2015), die zu wissensorientierter Argumentation bei Kindern forschen. Dieser Forschungszweig untersucht in der Regel Kinder ab mindestens dem Grundschulalter und arbeitet mit experimentellen oder doch zumindest elizitierten Daten, häufig unter Beteiligung von Erwachsenen in der Rolle der Studienleiter/innen. Dies findet sich beispielsweise auch bei Pontecorvo/Arcidiacono (2010:22), die in ihren Daten einen weiteren für uns wichtigen Aspekt identifizieren: In Argumentation, hier untersucht anhand der Diskussion eines Märchens, konstruieren Kindern teilweise Oppositionen, um diese dann selbst aufzulösen. Solche Fälle finden wir auch in unseren Daten. Clark et al. (2003) fassen in einer Studie zum kollaborativen Begründen von Viertklässler/inne/ $\mathrm{n}$ in der Diskussion eines Buches Argumentation als Weg, sich einem Gegenstand, einem Wissensbereich zu nähern. 
Diese epistemische Dimension des Argumentierens hat auch der Mathematiker und Mathematik-Didaktiker Krummheuer (1995) bearbeitet. Er untersucht, wie Kinder argumentieren, wenn sie mathematische Probleme lösen. Solche argumentativen Praktiken rahmt er als Praktiken der Wissensgenerierung und -etablierung und nennt als das zentrale Merkmal von Argumentation „Geltung etablieren": "If one or several participants accomplish an assertion like , 4 x 10 $=10 \times 4^{\prime}$ they do not only produce a sentence; rather they make a declaration inasmuch as they claim such a statement to be valid. By proposing it they are not only indicating that they try to act rationally, but also that they could establish this claim in more detail, if desired. Usually, these techniques or methods of establishing the claim or statement are called an argumentation" (ebd.:232, Hervorhebung im Original). Diese Etablierung von Geltung findet sich auch in der Studie von Pontecorvo/Arcidiacono (2010). Die Autorinnen zeigen, dass Kinder in Rahmen von Argumentation Kategorien erarbeiten und verhandeln (ebd.:22).

Ausgehend von dem Forschungsdesiderat, dass „little or nothing is known about how young children justify and build theories of the world together with same-age peers through naturally occuring interaction“ (115), untersuchen Kyratzis/ Ross/Koymen (2010) „Rechtfertigungen“ (justifications) in Kind-Kind-Interaktion. Sie unterscheiden in diesem Zusammenhang zwischen validierend und oppositional motivierten Kontexten dieser Rechtfertigungen. Es ist genau diese Schnittstelle, diese doppelte Funktion von Begründungen, die wir untersuchen.

Durch eine solche Schwerpunktsetzung wird auch die Unterscheidung von Erklären und Argumentieren relevant. Nach Morek (2012) unterscheiden sie sich durch die epistemische Haltung, die die Beteiligten zu ihren Aussagen einnehmen: Beim Erklären ist sie durch Gewissheit gekennzeichnet, beim Argumentieren durch Verhandelbarkeit (so auch Heller/Krah 2015:5). Hier sei allerdings darauf verwiesen, dass die klare Bestimmung von Äußerungen als Argumentation oder Erklären/Erklären-warum nicht immer möglich ist und Äußerungen durchaus beide Funktionen vereinen können (Deppermann 2003:14). Antaki (1994) nimmt für das Geben von Accounts (Erklärungen/Gründe) eine konstruktive Funktion im Sinne der Konstruktion und Aufrechterhaltung einer moral order in Goffmans Sinn an (113). „Proponents of treating argument and explanation as both being essentially activities of claim-backing acknowledge, then, that although they are conceptually distinct at the extremes, they are not easily separable in the grey area in the center" (Antaki 1994:142). In diesem Sinne verstehen wir das Verhältnis von Erklären und Argumentieren eher als empirische Frage denn als gegebene Unterscheidung. Unsere Vorannahme ist, dass Erklären und Argumentieren in der Praxis nicht immer klar unterscheidbar sind und dass auch Erklärungen eine argumentative Funktion haben, indem sie argumentative Ressourcen in Form von „kollektiv Geltendem“(W. Klein 1980) bereitstellen.

Zusammenfassend für die Argumentation von Vorschulkindern in KindKind-Kommunikation lässt sich festhalten, dass, obwohl die Entstehung der Fähig- 
keit zu begründen relativ übereinstimmend etwa im Alter von zwei Jahren angesetzt wird, die Funktion des Argumentierens sich vermutlich doch deutlich wandelt. Dabei zeigen die vorhandenen Studien (allerdings auf sehr schmaler Datenbasis anhand weniger Kinder und selten in Kind-Kind-Kommunikation), dass Kinder zu Beginn ihrer Entwicklung Begründungen nur in bestimmten Kontexten verwenden, wobei sie auf die Hilfe erwachsener Bezugspersonen angewiesen sind, dass Kinder im Vorschulalter die häufigsten sprachlichen Begründungsmarkierungen bereits beherrschen, dass spielende Kinder im Alter von drei bis sechs Jahren Begründungen oft nicht aus Meinungsverschiedenheiten heraus geben, sondern selbstinitiiert und offensichtlich um Aussagen und Handlungen der kindlichen Partner/innen zu stützen (Kyratzis et al. 2010).

\section{Stimmliche und körperliche Beredsamkeit}

Eine rhetorische Untersuchung zur Etablierung von Geltung in natürlicher Interaktion fokussiert auch auf die actio, also auf die performativen Aspekte des rhetorischen Handelns. Hier wird in der Rhetoriktheorie neben dem Inhaltsaspekt das Wie, die körperliche Beredsamkeit, bedeutsam. Es ist ein alter rhetorischer Grundsatz, dass die Wirkung gesprochener Äußerungen nicht nur vom strukturell-sprachlichen, sondern auch vom stimmlich-artikulatorischen und körperlichen Ausdruck abhängt, denn beide Ausdrucksebenen liefern Hinweise darauf, wie etwas, was ein/e Sprecher/in sagt, gemeint ist. Seit der Antike umfasst die rednerische Praxis als Teil der rhetorices partes neben den sprachlichen immer auch stimmlich-artikulatorische Gestaltungsmittel (Stimme, Lautheit, Sprechgeschwindigkeit, Rhythmus, Pausen u.a.m.), die sog. figurae vocis, und körperliche Gestaltungsmittel (Haltung, Gestik, Mimik, Atemtechnik), den sog. motus corporis (vgl. Rhetoric ad Herennium 1998, Cicero 2007, Quintilian 2011).

Damit ist rhetorisch formuliert, was auch für die linguistischen und hier insbesondere gesprächsanalytischen Zugriffe auf Interaktion relevant ist: die Multimodalität von Äußerungen und in diesem Rahmen auch die Bedeutung von stimmlichen und anderen körperlichen Parametern. In der rhetorischen Forschung findet sich dieses neu gestiegene Interesse innerhalb der Sound Studies und der Rhetorik unter der Überschrift sonic persuasion (Goodale 2011, Gunn et al. 2013, Gunn 2007). Aus sprechwissenschaftlicher Sicht lässt sich die Integration der körperlichen Beredsamkeit in die Analyse mit dem Begriff des "stimmlich-artikulatorischen Ausdrucks“ fassen (z.B. Stock 1991, Bose 2003 und 2010). Der Begriff beschreibt situations- und stimmungsadäquat konventionalisierte Gestaltungsweisen im Sprechschall, mit denen Sprecher u.a. Rollen-, Gruppen-, Handlungsmuster realisieren. Stimmlich-artikulatorischer Ausdruck ist mit der sprachlichen Ebene des Sprechens eng verwoben und stellt einen Merkmalskomplex aus stimmlich-melodischen, temporalen, dynamischen und artikulatorischen Parametern dar, die an auditiv wahrnehmbaren physiolo- 
gisch-akustischen Grundlagen der Stimmproduktion und Artikulation orientiert sind (Sprechtonhöhe, Stimmklang, Lautheit, Sprechgeschwindigkeit, Akzentuierung, Gliederung, Artikulation u.a.m.). Analog wird der situations- und stimmungsspezifische Umgang mit Mimik, Gestik, Körperhaltungen und -bewegungen (bis hin zum „Sich (Ver-)Kleiden“) als „Körperausdruck“ betrachtet. Hier lassen sich Verbindungen ziehen zu Konzepten wie den Körpertechniken (Mauss 1934) und Körperpraktiken (z.B. Frieling 2003) sowie der Verkörperung- bzw. Embodiment-Forschung (stellvertretend Johnson 1992, Csordas 1994, Ziemke/Zlatev/Frank 2007), die alle davon ausgehen, dass der „Körper als Medium und Material für Zeichenbildung verwendet“ wird (Fischer-Lichte 2001:16), „daß Kognition als embodied activity zu verstehen und zu untersuchen, daß der Geist immer verkörpert ist" (20). Produktiv ist der Ausdrucksbegriff, weil er nicht nur auf das Individuum verweist, das seinen Empfindungen willkürlich oder unwillkürlich Ausdruck gibt, sondern auch auf den Adressaten, der Ausdrucksformen wahrnimmt und interpretiert und bei dem ebenfalls Empfindungen erregt werden, und damit auf den Interaktionsprozess; Ausdruck ist immer Ausdruck von jemandem für jemanden in einer konkreten Situation.

Hier lässt sich ein Bezug zur Ontogenese herstellen, genauer zur kindlichen Protorhetorik (Antos 1985): Kinder kommen zur Sprache über den stimmlich-artikulatorischen Emotionsausdruck in Protokonversationen mit erwachsenen Bezugspersonen (Papoušek 1994, Tomasello 2002). Dadurch können schon sehr kleine Kinder stimmlich-artikulatorische und körperliche Ausdrucksfähigkeiten für die Behauptung und Durchsetzung von Geltungsansprüchen nutzen (Garvey 1984). Kleine Kinder verfügen über stimmlich-artikulatorische und körperliche Ausdrucksfähigkeiten, die ihren sprachlichen gleich kommen oder diese überschreiten (Garvey 1984, Bose 2003). Bei der Durchsetzung kommunikativer Ziele besitzen stimmlich-artikulatorische und körperliche Ausdrucksweisen für kleine Kinder offensichtlich denselben kommunikativen Rang wie verbale Handlungsfolgen (J. Klein 1985). Stimmlich-artikulatorische und körperliche Ausdrucksmittel, die die Kinder bereits vorsprachlich etabliert haben, setzen sie auch für die Sicherung der Konsensualität und Kooperativität in Kommunikationen mit Peers ein. So spielen z.B. in konsensuellen Interaktionen Rhythmus und Symmetrie eine besondere Rolle (Bose 2003). Wenn also kindliche (protorhetorische) Praktiken zur Etablierung von Geltung untersucht werden sollen, sind auch stimmlich-artikulatorische und körperliche Ausdrucksformen zu berücksichtigen.

Wir beobachten in unseren Daten (s. unten), dass Begründungen oft in harmonischen (Spiel-) Situationen auftreten, in denen die Kinder den Spielrahmen aufrecht halten und die fiktive Spielwelt ausbauen wollen. In diesen Situationen begründen die Kinder oft nicht aus Meinungsverschiedenheiten heraus, also nicht zur Bearbeitung von Dissens, sondern selbstinitiiert kooperativ/kollaborativ/ko-konstruktiv. Offensichtlich wollen sie Vorschläge und Handlungen des Spielpartners stützen und Konsens über eingebrachte Spielvorschläge ausstellen/ demonstrieren (so auch Birmele et al. 2007, Doury 2012, Arendt 2014). 


\section{Exemplarische Analysen}

Abschließend präsentieren wir drei spielbezogene Begründungssequenzen aus dem Korpus A.

Sie stammen aus einem sog. Requisitenspiel (Komor 2010:67ff.) zweier vierjähriger Mädchen mit Lego-Duplo-Bausteinen und -Puppen, das sowohl Anteile eines Bauspiels auch eines Rollenspiels aufweist. Dieses Spiel wurde bereits in Bose (2003) im Hinblick auf spielbezogene Formen und Funktionen des stimmlich-artikulatorischen Ausdrucks analysiert, vgl. z.B. den sog. Erzählton zur Kommentierung von Spielhandlungen aus einer Erzählerperspektive (ebd.:296ff.) oder die gegenseitige Iteration stimmlich-artikulatorischer Gestaltungen zur Demonstration besonderer Vertrautheit zwischen den Kindern (352ff.).

Die beiden Kinder (Siglen: Kind 1 = K1, Kind 2 = K2) haben auf dem Fußboden eine Legowohnung gebaut und platzieren nun die Legopuppen darin. Sie kommentieren ihre Spielhandlungen, schlüpfen gelegentlich in die jeweiligen Puppen-Identitäten und führen in den Spielrollen Gespräche. Eingebettet in kooperativ-kollaborative Spielhandlungen sind immer wieder verbale, paraverbale und nonverbale Praktiken zur Etablierung von Geltung auszumachen. Es handelt sich um sehr kurze Passagen, das ist typisch für viele Begründungssequenzen innerhalb dieses Spiels.

Die Transkription folgt GAT2 (2009), allerdings wird nur eine Akzentstufe vermerkt (Großschreibung der akzentuierten Silbe), außerdem wird am Ende von Sprecheinheiten vermerkt, ob sie als inhaltlich-prosodisch abgeschlossen $(\|)$ oder nicht-abgeschlossen (|) interpretiert werden (zum Konzept der Abgeschlossenheit vgl. stellvertretend Stock 1996). Die Abbildungen zu den Beispielen stammen aus den Videoaufnahmen. Alle Rechte dafür liegen bei den Autorinnen.

\subsection{Beispiel 1: „Restaurant“}
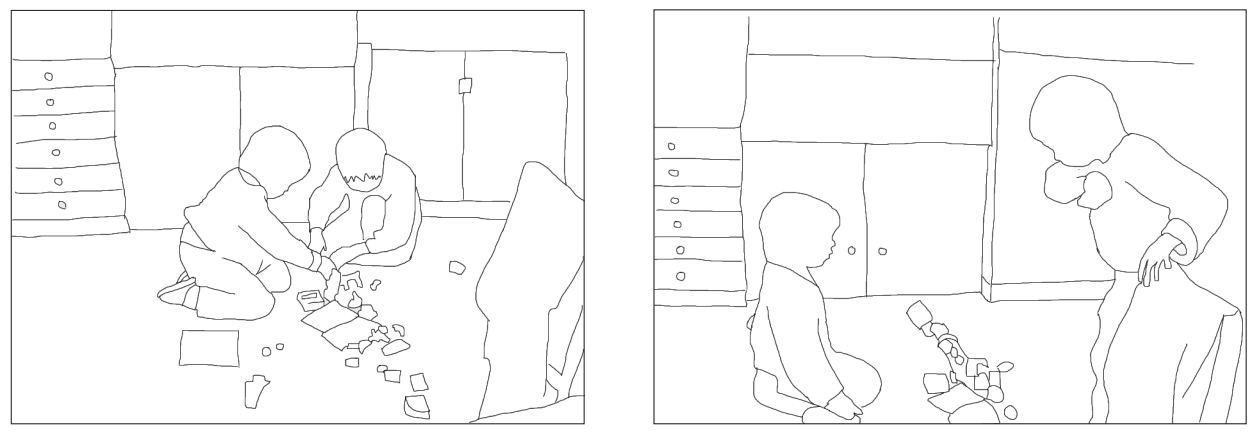


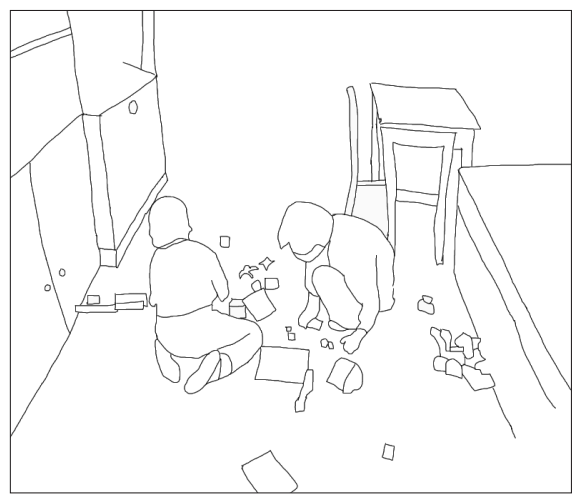

Die beiden Mädchen platzieren Legopuppen in der gebauten Lego-Wohnung (LW), konkret auf Bausteinen, die sie als Sitzbänke definieren. Die Kinder verwenden durchgängig einen rhythmisch-melodischen, langsamen Sprechgesang, den sie für gewöhnlich in intensiv-harmonischen Spielpassagen als Erzählmuster nutzen (Bose 2003:296ff.). Diesen konsens-betonenden Sprechgesang behalten sie auch konsequent bei, während sie einen lokalen Dissens bearbeiten.

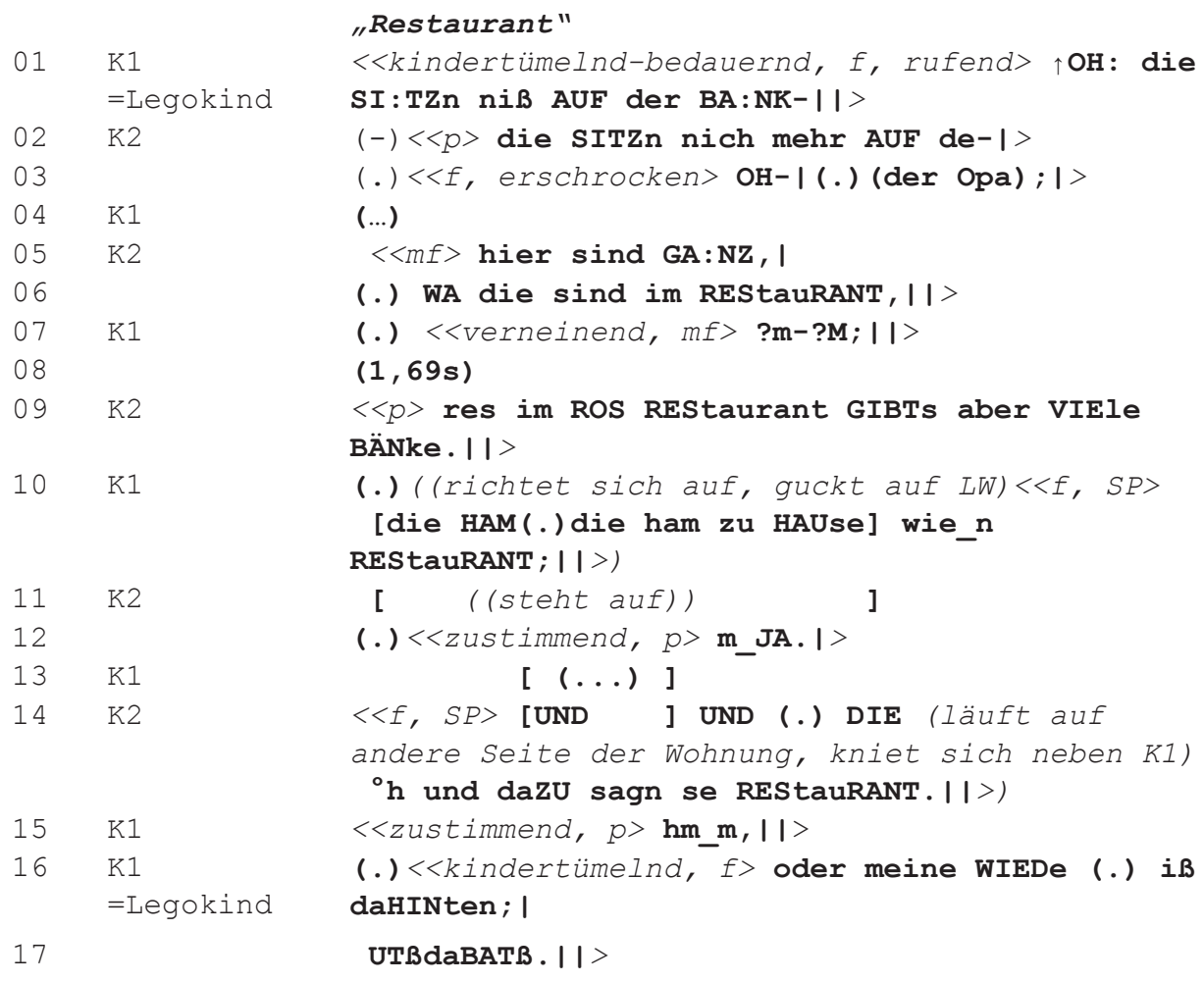




$\begin{array}{lll}18 & \text { K2 } & (-)<<\text { indertümelnd, f> UTBdaBATB, II> } \\ \text { =Legokind } & & \\ 19 & \text { K1 } & \text { (.) }<<\text { kindertümelnd, p> ham die geßAGT-||> } \\ 20 & \text { K2 } & \text { (.) }<<p>\text { geSA:GT-|| }>\end{array}$

In Z01 stellt Kind 1 in der fiktiven Identität eines ,kleinen Legokindes“ in kindertümelnd-bedauerndem stimmlich-artikulatorischem Ausdrucksmuster (Bose 2003: 233ff.) fest, dass einige Puppen nicht mehr auf der Bank sitzen. In Z02 wiederholt Kind 2 diese Äußerung leise, unterbricht sich dann selbst und kommentiert mit erstaunt-erschrockenem stimmlich-artikulatorischen Ausdruck, dass die Opa-Puppe gerade von der Bank gefallen ist (Z03). Die darauffolgende Äußerung von Kind 1 in Z04 ist nichtzu verstehen. In Z05 setzt Kind 2 an: hier sind GA:NZ,| (vermutlich „...viele Bänke“), führt diese Feststellung aber nicht zu Ende, sondern schlägt vor, dass sich die Puppen im Restaurant befinden: (.) WA die sind im REStauRANT, || (Z06). Diesen Vorschlag zum Szenenwechsel von Wohnung zu Restaurant spricht es gespannter, lauter und deutlicher als bisher und leitet ihn mit der Zustimmung heischenden Partikel wa ein, hebt ihn also sprachlich-sprecherisch heraus. Kind 1 lehnt den Vorschlag jedoch nonverbal-vokal ab (Z07) und markiert damit einen Dissens.

Es folgt eine überlange Pause (1,69 s). Pausen solcher Länge sind in diesem Korpus selten und zeigen eine Gesprächskrise an: Das Spiel „gefriert“, die Kinder scheinen quasi ratlos und oft setzen sie danach sprachliche und vor allem stimmlich-artikulatorische Deeskalationsstrategien ein, um das Spiel nicht zu gefährden (Bose 2003:194ff.). Kind 2 begründet und verteidigt nun seinen Spielvorschlag (Z09) leiser als vorher: res im ROS REStaurant GIBTs aber VIEle BÄNke.||. Es nutzt dafür den Teil-Ganzes-Topos in der trugschlüssigen Variante des modus ponens: Wenn ein Teil eines Restaurants vorhanden ist, nämlich ganz viele Bänke, dann ist alles ein Restaurant. Kind 1 reagiert mit einem (unmarkierten) Kompromiss: (.) die HAM (.) die ham zu HAUse wie n REStauRANT; | (Z10) und nutzt ebenfalls den Topos der Ähnlichkeit: Wenn es zu Hause (in der Legowohnung) aussieht wie in einem Restaurant (wegen der Bänke), dann haben die Puppen zu Hause wie ein Restaurant. Dabei lehnt sich Kind 1 zurück, geht also auf leichte Distanz zum Spielort (Legowohnung), richtet sich auf und spricht laut, gespannt und deutlich - es hebt also seinen Kompromissvorschlag innerhalb des etablierten konsensuellen Erzähltons heraus. Währenddessen steht Kind 2 auf (Z11), stimmt zu (Z12: $m \_J A$.|) und stärkt die Restaurant-Ähnlichkeit der Legowohnung aus der Perspektive der Puppen: Die Puppen sagen auch „Restaurant“ dazu (Z14: [UND] UND (.) DIE ${ }^{\circ} \mathrm{h}$ und daZU sagn se REStauRANT.||). Diese Äußerung markiert Kind 2 ähnlich wie vorher Kind 1 über erhöhte Sprechspannung, Lautheit und Deutlichkeit; außerdem auch nonverbal, indem es auf die andere Seite der Legowohnung läuft und sich neben Kind 1 kniet, also wieder körperliche Nähe zur Spielpartnerin herstellt.

Kind 1 stimmt nonverbal-vokal zu (Z15) und setzt das Spiel in der Identität einer kleinen Legopuppe kindertümelnd sprechend fort: oder meine WIEDe (.) i $\beta$ 
daHINten; $\mid$ (Z16). Es lispelt und benutzt ein kindertümelndes Fantasiewort (UTßdaBATß.\|; Z17), das von Kind 2 im selben Ausdrucksmuster wiederholt wird (Z18). Es entwickelt sich eine Folge von sprachlich und sprecherisch identischen Wiederholungen (Z19-20), die im Korpus Anzeichen besonderer Harmonie zwischen den Spielpartnerinnen sind (Bose 2003:350ff.).

Vor allem sprachlich und stimmlich-artikulatorisch zeigt sich das Bemühen der Kinder, trotz des Dissenses die grundsätzliche Kooperativität des Spiels nicht zu gefährden, sondern durch etablierte Konsens-demonstrierende Ausdrucksmuster (Erzählton, rhythmisiert-melodischer Singsang, Äußerungswiederholungen im selben stimmlich-artikulatorischen Ausdrucksmuster) weiterhin aufrecht zu erhalten. Die Argumentation ist hier klar enthymematisch, die Konklusion bleibt implizit. Zugleich zeigt sich ein wichtiges Merkmal enthymematischen Argumentierens: Jedes Kind kann die Konklusion einsetzen, die es für sich entwickelt, ohne dies wieder mit der Spielpartnerin diskutieren zu müssen. So können in diesem Fall sogar zwei widerstreitende Konklusionen als plausibel gesehen werden: Wenn die Puppen eine Wohnung wie ein Restaurant haben (Kind 1) und auch Restaurant dazu sagen (Kind 2), dann

1. ist das entweder hier (wie) ein Restaurant oder

2. es ist eben nicht wirklich (wie) ein Restaurant.

Mit dieser Vagheit legen die Kinder die Grundlage für weitere Kooperation.

In diesem Spielausschnitt handeln die Kinder also den Spielort aus (Sind die Puppen nun zu Hause oder im Restaurant?), indem sie gemeinsam die Eigenschaften diskutieren, die für den einen oder/und den anderen Ort sprechen. Damit verhandeln sie (implizit), was Ähnlichkeit ist. Als geltend ist zwischen beiden Kindern aber bereits etabliert, dass Ähnlichkeit überhaupt für Schlussfolgerungen heranzuziehen ist, sie nutzen Ähnlichkeit als formalen Topos.

\subsection{Beispiel 2: „Wackelig“}

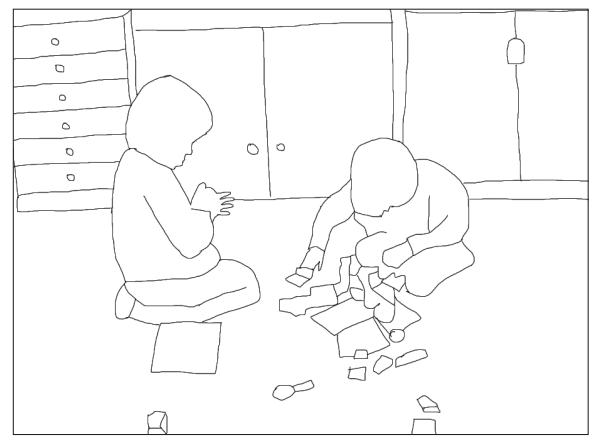

Auch in diesem Ausschnitt zwei Minuten später ist wieder durchgängig das rhythmisiert-melodische Singsang-Muster zu hören, das beide Kinder für die Ausstel- 
lung von Konsens und Harmonie verwenden. Jetzt versuchen die Mädchen, zwei Lego-Babies in einer Wiege unterzubringen.

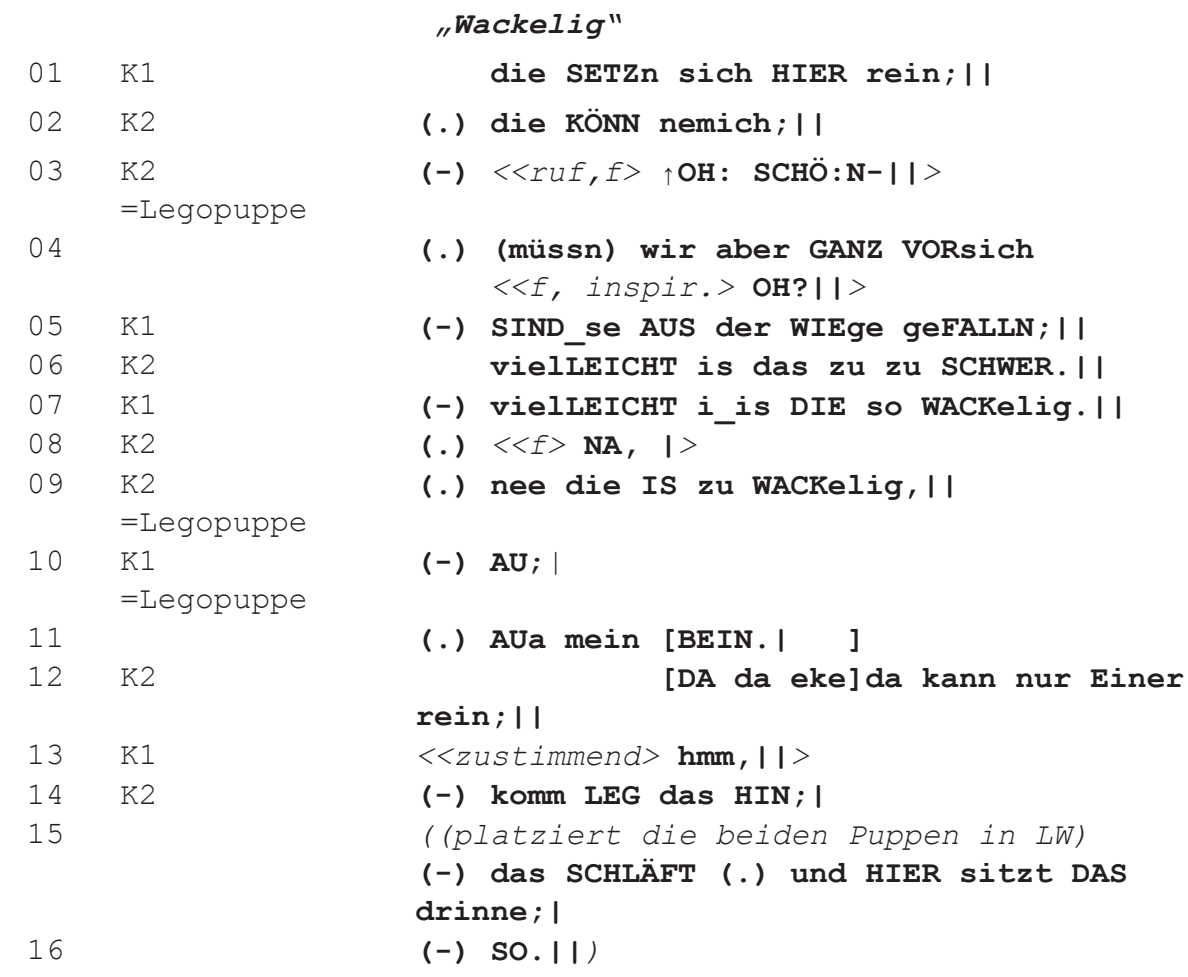

In dieser Passage gibt es keinerlei Dissensmarkierung. Die epistemische Funktion der Begründungssequenz besteht darin, dass die Kinder nach Erklärungen dafür suchen, warum etwas der Fall ist (warum die Puppen aus der Wiege gefallen sind: Z05). Die Kinder nennen zwei mögliche Gründe dafür:

1. Die Ursache ist das Lego-Baby - Kind 2 (Z06): vielLEICHT is das zu zu SCHWER. ||

2. Die Ursache ist die Konstruktion der Wiege - Kind 1 (Z07): (-) vielLEICHT i_is DIE so WACKelig. ||

Es gibt weder eine Infragestellung noch eine explizite Einigung. Allerdings übernimmt Kind 2 die Vermutung von Kind 1 (Z07: so WACKelig.||) insofern, als es sich kindertümelnd als Puppe fragt: nee die IS zu WACKelig, || (Z09). Die Klärung der Ursache hat eigentlich Konsequenzen fürs Weiterspielen. Aber die Schlussfolgerung, die Kind 2 in Z12 zieht ([DA da eke]da kann nur Einer rein;||), passt auf beide Begründungen: Da kann nämlich nur einer rein,

1. entweder weil beide Puppen zu schwer sind

2. oder weil die Wiege zu wacklig ist. 


\subsection{Beispiel 3: „Stuhllehne“}

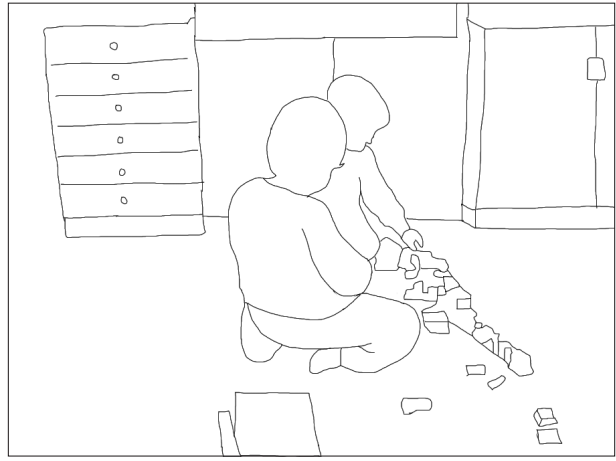

Zehn Minuten später suchen die beiden Mädchen Schlafplätze für weitere Legopuppen, konkret für einen Hund und eine Schnecke. Wieder kommentieren sie ihre Spielhandlungen, schlüpfen gelegentlich in die jeweiligen Puppen-Identitäten und führen Spielrollen-Dialoge.

$\begin{array}{ll}01 & \begin{array}{l}\text { K2 } \\ =\text { Legohund }\end{array} \\ 02 & \\ 03 & \text { K1 } \\ & \\ 04 & \text { K2 } \\ & =\text { Legomama } \\ 05 & \text { K1 } \\ 06 & \\ 07 & \end{array}$

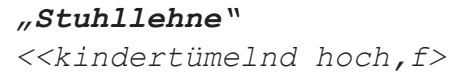

In dieser Sequenz geben die Kinder - zumindest für die außenstehenden erwachsenen Beobachterinnen - überraschenderweise und quasi nebenher Begründungen, ohne dass diese eingefordert werden und ohne dass sprachliche, stimmlich-artikulatorische oder körperliche Indizien für einen lokalen Dissens oder eine Spannung bzw. Gereiztheit zwischen den Kindern vorliegen. Dies wird in Zeile $01-03$ besonders deutlich, weil die Kinder hier gemeinsam einen „umgekehrten" Syllogismus aus conclusio, propositio minor und major produzieren und dafür den Topos der Analogie bzw. des Vergleichs nutzen: Kind 2 schlüpft in die Identität des kleinen Legohundes und spricht rollengemäß in kindertümelndem stimmlich-artikulatorischem Ausdrucksmuster (Bose 2003:230ff.). Es macht einen Spielvorschlag: und (.) dann KOMM wir SCHLAfen auf der STUHLlehne;| (Z01). Diese Äußerung fungiert als conclusio: Sie wird in keiner Weise als strittig 
markiert, dennoch aber wird sie von beiden Kindern gemeinsam gestützt. Zunächst begründet Kind 2 selbst seinen Vorschlag - immer noch kindertümelnd als Legohund sprechend (Z02): (.) wir sind doch TIEre;||. Diese Begründung stützt Kind 1 aus der Erzählerperspektive mit der allgemeinen Prämisse: (-) die TIEre SCHLAfen auf der STUHLlehne;|| (Z03). Dann schlüpft Kind 2 in die fiktive Identität der „Legomutter“ und wendet sich in mütterlichem stimmlich-artikulatorischem Ausdrucksmuster (Bose 2003, 223ff.) an die Puppe eines Legojungen: (.) $d u$ GRO:ßer-|| (Z04). Danach verfolgt Kind 1 das Schlafplatz-Thema weiter, indem es fragt: (-) was schläft HIER; | (Z05). Es schlägt vor: (.) $<<f>$ das KANN ja auch im STEHN schlafen-| (Z06) und begründet den Spielvorschlag unaufgefordert mit einer Analogie: DIEse SCHLAfen ja beide AUCH im stehn; || (Z07).

Wenn auch längst nicht immer so elaboriert wie mit diesem „umgekehrten“ Syllogismus aus conclusio, propositio minor und major, setzen Vorschulkinder im Spiel doch sehr oft Begründungshandeln nicht zur Dissensbearbeitung ein, sondern kooperativ-kollaborativ zur gemeinsamen Erschaffung von Spielwelten und zur Ausstellung von Konsens über eingebrachte Spielvorschläge.

\section{Ausblick}

Unsere Beispiele zeigen, dass Argumentation als Begründungshandeln in der Kind-Kind-Kommunikation im Vorschulalter auch eingesetzt wird, um Geltung $\mathrm{zu}$ etablieren, und damit eine epistemische Funktion hat. Möglicherweise, das werden wir in weiteren Analysen untersuchen, dominiert diese Funktion in bestimmten Altersspannen und Situationen über die Funktion der Dissensbearbeitung. Die Etablierung von Geltung impliziert, dass hier Ressourcen für kommende Argumentation aufgebaut werden. Was jetzt als geltend akzeptiert wird, kann später zur Stützung strittiger Aussagen herangezogen werden. Damit versteht sich unsere Forschung auch als ein Beitrag zur Ethnographie der Argumentation und dem Interesse daran, wie eine Aussage überhaupt den Status einer Prämisse erlangen kann. Die Ergebnisse können darüber Aufschluss geben, wie Kinder untereinander aushandeln, was Geltung hat, damit als geteiltes Wissen gelten kann und so als Ressource innerhalb von Dissens-orientierten Argumentationen dienen kann.

\section{Literatur}

ANDRESEN Helga, 2002, Interaktion, Sprache und Spiel. Zur Funktion des Rollenspiels für die Sprachentwicklung im Vorschulalter, Tübingen.

ANDRESEN Helga, 2005, Vom Sprechen zum Schreiben. Sprachentwicklung zwischen dem vierten und siebten Lebensjahr, Stuttgart/London.

ANTAKI Charles, 1994, Explaining and Arguing. The Social Organisation of Accounts, London. 
ANTOS Gerd, 1985, Proto-Rhetorik. Zur Ontogenese rhetorischer Fähigkeiten, in: Dyck J./Jens W./ Ueding G. (Hrsg.), Rhetorik. Ein internationales Jahrbuch. Bd. 4, Tübingen, S. 7-28.

ARENDT Birte, 2014, Konfliktbearbeitungen von Kindergartenkindern - verbale resp. argumentative und nonverbale Muster, in: Mémoires de la Société Néophilologique de Helsinki 92, S. 21-34.

ARENDT Birte, 2015, Kindergartenkinder argumentieren. Peer-Gespräche als Erwerbskontext, in: Mitteilungen des Deutschen Germanistenverbandes 62/1, S. 21-33.

ARENDT Birte / HELLER Vivian / KRAH Antje (Hrsg.), 2015, Kinder argumentieren. Interaktive Erwerbskontexte und -mechanismen, in: Mitteilungen des Deutschen Germanistenverbandes 62, S. 1.

Auctor ad Herennium,1998, Rhetoric ad Herennium, Düsseldorf/Zürich.

Birmele Karin / Grasser Barbara / Guckelsberger Susanne / Komor Anna / Trautmann Caroline, 2007, Sprachliche Kooperation im kindlichen Requisitenspiel, in: Redder A. (Hrsg.), Diskurse und Texte. Festschrift für Konrad Ehlich zum 65. Geburtstag, Tübingen, S. $459-472$.

Bose Ines, 2003, „doch da sin ja nur muster.“ Kindlicher Sprechausdruck im sozialen Rollenspiel, Frankfurt a. M.

Bose Ines, 2010, Stimmlich-artikulatorischer Ausdruck und Sprache, in: Deppermann A./Linke A. (Hrsg.), Sprache intermedial. Stimme und Schrift, Bild und Ton (= Jahrbuch des IDS 2009), Berlin/New York, S. 29-68.

Bose Ines / HANNKEN-ILLJES Kati, 2012, Protorhetorik, in: Ueding G. (Hrsg.), Historisches Wörterbuch der Rhetorik. Bd. 10: Nachträge A-Z, Tübingen, S. 966-975.

BRUMARK Asa, 2007, Argumentation at the Swedish family dinner table, in: van Eemeren F./Blair J.A./ Willard C.A./Garssen B. (Hrsg.), Proceedings of the Sixth Conference of the International Society for the Study of Argumentation. A-K, Amsterdam, S. 169-178.

BRUMARK Asa, 2008, "Eat your Hamburger!" - "No, I don't want to!" Argumentation and Argumentative Development in the Context of Dinner Conversation in Twenty Swedish Families, in: Argumentation 22, S. 251-271.

BRÜNE Martin / BRÜNE-COHRS Ute, 2006, Theory of mind - evolution, ontogeny, brain mechanisms and psychopathology, in: Neuroscience and Biobehavioral Reviews 30, S. 437-455.

BURKE Kenneth, 1969, A Rhetoric of Motives, Berkeley.

Clark Ann-Marie / Anderson Richard C. / KuO Li-jen / Il-HeE Kim / ArCHOdidou Anthi / NGUYEN-JAHIEL Kim, 2003, Collaborative reasoning: Expanding ways for children to talk and think in school, in: Educational Psychology Review 15, S. 181-198.

CICERO Marcus Tullius, 2007, De Oratore - Über den Redner, Düsseldorf/Zürich.

CONLEY Thomas, 2000, What counts as topos in contemporary research, in: Schirren T./Ueding G. (Hrsg.), Topik und Rhetorik, Tübingen, S. 579-586.

Crowell Amanda / KuHn Deanna, 2014, Developing Dialogic Argumentation Skills. A 3-Year Intervention Study, in: Journal of Cognition and Development 15/2, S. $363-381$.

CsORDAS Thomas J. (Hrsg.), 1994, Embodiment and Experience. The Existential Ground of Culture and Self, Cambridge.

DEPPERMANN Arnulf, 2003, Desiderata einer gesprächsanalytischen Argumentationsforschung, in: Deppermann A./Hartung M. (Hrsg.), Argumentieren in Gesprächen. Gesprächsanalytische Studien, Tübingen, S. 10-26.

DouRY Marianne 2012, Preaching to the Converted. Why Argue When Everyone Agrees? in: Argumentation 26, S. $99-114$.

EHLICH Konrad / BREDEL Ursula / REICH Hans (Hrsg.), 2008a, Referenzrahmen zur altersspezifischen Sprachaneignung. Referenzrahmen, Bildungsforschung. Bd. 29/I, Bonn (http://www. bmbf.de/pub/bildungsforschung_bd_neunundzwanzig_eins.pdf).

EHLICH Konrad / BREDEL Ursula / REICH Hans (Hrsg.), 2008b, Referenzrahmen zur altersspezifischen Sprachaneignung. Forschungsgrundlagen, Bildungsforschung. Bd. 29/II, Bonn (http:// www.bmbf.de/pub/bildungsforschung_bd_neunundzwanzig_zwei.pdf). 
FRIELING Kirsten O., 2003, Ausdruck macht Eindruck. Bürgerliche Körperpraktiken in sozialer Kommunikation um 1800, Frankfurt a. M.

FISCHER-LICHTE Erika, 2000, Theatralität und Inszenierung, in: Fischer-Lichte E./Pflug I. (Hrsg.), Inszenierung von Authentizität, Tübingen/Basel, S. 11-27.

GARVEY Catherine, 1984, Children's Talk, London.

GoodALE Greg, 2011, Sonic Persuasion. Reading Sound in the Recorded Age, Urbana.

GUNN Joshua, 2007, Gimme Some Tongue (on Recovering Speech), in: Quarterly Journal of Speech 93/3, S. 361-364.

Gunn Joshua / GoodAle Greg / Hall Mirko / Eberly Rosa, 2013, Ausculating Again: Rhetoric and Sound Studies, in: Rhetoric Society Quarterly 43/5, S. 475-489.

HABERMAS Jürgen, 1995, Theorie des kommunikativen Handelns. Bd. 1 Handlungsrationalität und gesellschaftliche Rationalisierung, Frankfurt a. M.

HANNKEN-ILLJES Kati, 2004, Gute Gründe geben, Frankfurt a. M.

HANNKEN-IllJes Kati / Holden Livia / KozIN Alexander / ScheFfER Thomas, 2007, Trial and Error. Failing and Learning in Criminal Proceedings, in: International Journal for the Semiotics of Law 20, S. $159-190$.

HELLER Vivian, 2011, Die Herstellung kommunikativer Kontexte in familialen Tischgesprächen, in: Birkner K./Meer D. (Hrsg.), Institutionalisierter Alltag: Mündlichkeit und Schriftlichkeit in unterschiedlichen Praxisfeldern, Mannheim, S. 92-116.

HELLER Vivian / KRAH Antje, 2015, Wie Eltern und Kinder argumentieren. Interaktionsmuster und ihr erwerbssupportives Potenzial im längsschnittlichen Vergleich, in: Mitteilungen des Deutschen Germanistenverbandes 62/1, S. 5-20.

JohnStONE Henry Jr., 1965, Some Reflections on Argumentation, in: Natanson M./Johnstone H. Jr. (Hrsg.), Philosophy, Rhetoric, and Argumentation, University Park, S. 1 -9.

KLEIN Josef, 1985, Vorstufen der Fähigkeit zu BEGRÜNDEN bei knapp 2-jährigen Kindern, in: Kopperschmidt J./Schanze H. (Hrsg.), Argumente. Argumentation, München, S. 261 - 271.

KLEIN Wolfgang, 1980, Argumentation und Argument, in: Zeitschrift für Literaturwissenschaft und Linguistik 10(38/39), S. $9-57$.

KNOBLAUCH Hubert, 2000, Topik und Soziologie. Von der sozialen zur kommunikativen Topik, in: Schirren T./Ueding G. (Hrsg.), Topik und Rhetorik, Tübingen, S. 651-668.

KOMOR Anna, 2010, Miteinander kommunizieren - Kinder unter sich. Eine empirische diskursanalystische Untersuchung zur Ausbildung kindlicher Kommunikationsfähigkeit, Münster.

KOPPERSCHMIDT Josef, 1989, Methodik der Argumentationsanalyse, Stuttgart.

KRAFT Barbara / MENG Katharina, 2007, Streit im Kindergarten. Eine Diskursanalyse, in: Redder A. (Hrsg.), Diskurse und Texte, Tübingen, S. 439-457.

KRUMMHEUER Götz, 1995, The Ethnography of Argumentation, in: Cobb P./Bauersfeld H. (Hrsg.), The Emergence of Mathematical Meaning: Interaction in Classroom Cultures, Hillsdale, S. $229-269$.

KUHN Deanna / UdELL Wadiya, 2003, The Development of Argument Skills, in: Child Development $74 / 5$, S. $1245-1260$.

KYRATZIS Amy Shuqum / Ross Tamara / KoYmEn S. Bahar, 2010, Validating Justifications in Preschool Girls' and Boys' Friendship Group Talk Implications for Linguistic and socio-cognitive Development, in: Journal of Child Language 37/1, S. 115-144.

MAuss Marcel, 1934/1974, Les techniques du corps, in: Journal de Psychologie 32(3-4). Die Techniken des Körpers, in: Mauss, M., Soziologie und Anthropologie, Bd. 2, München 19, 4, S. $19-22$.

MiLler Max, 1980, Zur Ontogenese moralischer Argumentationen, in: Zeitschrift für Literaturwissenschaft und Linguistik 10(38/39), S. 58-108.

MoReK Miriam, 2012, Kinder erklären. Interaktionen in Familie und Unterricht im Vergleich, Tübingen. 
Muller-Mirza Nathalie / Perret-Clermont Anne-Nelly / TARTAS Valerie / IANNACONNE Antonio, 2009, Psychosocial Processes in Argumentation, in: Muller-Mirza N./Perret-Clermont A.-N. (Hrsg.), Argumentation and Education. Theoretical Foundations and Practices, Berlin, S. $67-90$.

NELSON Katherine, 1996, Language in Cognitive Development, Cambridge.

PAPOUŠEK Mechthild, 1994, Vom ersten Schrei zum ersten Wort: Anfänge der Sprachentwicklung in der vorsprachlichen Kommunikation, Bern.

Perret-Clermont Anne-Nelly / Arcidiacono Francesco / BreuX Stéphanie / Greco Sarah / MisereZ-CAPEROS Céline, 2015, Knowledge-oriented Argumentation in Children, in: Garssen B.J./Godden D./Mitchell G./Snoeck Henkemans A.F. (Hrsg.), Proceedings of the 8th International Conference of the International Society for the Study of Argumentation, Amsterdam, S. $1118-1126$.

PonTECORVo Clotilde / ARCIDIACONO Francesco, 2010, Development of Reasoning through Arguing in Young Children, in: Культурно-Историческая Психология/Cultural-Historical Psychology 4, S. 19-29.

PRIOR Paul, 2005, Toward the Ethnography of Argumentation, in: Text 25/1, S. $129-144$.

QUASTHOFF Uta / KERN Friederike, 2007, Familiale Interaktionsmuster und kindliche Diskursfähigkeit, in: Hausendorf H. (Hrsg.), Gespräch als Prozess, Tübingen, S. 277-305.

QUASTHOFF Uta / KRAH Antje, 2015, Familiale Kommunikation als Spracherwerbsressource: Das Beispiel argumentativer Kompetenzen, in: Neuland E. (Hrsg.), Sprache der Generationen, Frankfurt a. M., S. 127-144.

Quintillian Marcus Fabius, 2011, Ausbildung des Redners, Darmstadt.

RÖSKA-HARDY Louise, 2011, Der Erwerb der Theory of Mind-Fähigkeit. Entwicklung, Interaktion und Sprache, in: Hoffmann L./Leimbrink K./Quasthoff U. (Hrsg.), Die Matrix der menschlichen Entwicklung, Berlin, S. 96-142.

SANDOVAL William A. / ÇAM Aylin, 2011, Elementary Children's Judgements of the Epistemic Status of Sources of Justification, in: Science Education 95/3, S. $383-408$.

SCHWARZE Cordula, 2010, Formen und Funktionen von Topoi im Gespräch, Frankfurt a. M.

SELting Margret / Auer Peter / BARTH-Weingarten Dagmar / Bergmann Jörg / BERGMANn Pia / BIRKNER Karin / COUPER-KUHLEn Elizabeth / DEPPERMANN Arnulf / GILLES Peter / GÜNTHNER Susanne / HARTUNG Martin / KERN Friederike / MERTZLUFFT Christine / MEYER Christian / MoreK Miriam / OberZaucher Frank / Peters Jörg / Quasthoff Uta / SchÜtTe Wilfried / StUKENBROCK Anja / UHMANN Susanne, 2009, Gesprächsanalytisches Transkriptionssystem 2 (GAT 2), in: Gesprächsforschung - Online-Zeitschrift zur verbalen Interaktion, Ausgabe 10, S. $353-402$ (www.gespraechsforschung-ozs.de).

SPRANZ-FogASY Thomas, 2003, Alles argumentieren oder was? - Zur Konstitution von Argumentation in Gesprächen, in: Deppermann A./Hartung M. (Hrsg.), Argumentieren in Gesprächen. Gesprächsanalytische Studien, Tübingen, S. 27-39.

STOCK Eberhard (in Zusammenarbeit mit SUTTNER Jutta), 1991, Wirkungen des Stimm- und Sprechausdrucks, in: Krech E.-M./Richter G./Stock E./Suttner J., Sprechwirkung. Grundfragen, Methoden und Ergebnisse ihrer Erforschung, Berlin (DDR), S. 59-142.

STосK Eberhard, 1996, Deutsche Intonation, Leipzig u.a.

TOMASEllo Michael, 2002, Die kulturelle Entwicklung des menschlichen Denkens. Zur Evolution der Kognition, Frankfurt a. M.

VöLZING Paul-Ludwig, 1982, Kinder argumentieren. Die Ontogenese argumentativer Fähigkeiten, Paderborn/München/Wien/Zürich.

YÁNÉZ Christian Santibánez, 2015, Steps Towards an Evolutionary Account of Argumentative Competence, in: Informal Logic 35/2, S. $168-183$.

ZiEMKE Tom / ZLATEV Jordan / FrAnK Rosalyn (Hrsg.), 2007, Body, Language and the Mind. Vol. 1: Embodiment, Berlin/New York. 